\title{
The Determination of Spin-Rotation and Spin-Spin Interaction Constants from the Microwave Spectrum of Methane
}

\author{
P. Wolf and W. Stahl \\ Abteilung Chemische Physik im Institut für Physikalische Chemie der Universität Kiel, FRG
}

Z. Naturforsch. 44a, 651-654 (1989); received April 21, 1989

High sensitivity and resolution of our microwave Fourier transform (MWFT) spectrometer and careful analysis of the measured transients enabled us to determine very accurate spin-rotation and spin-spin interaction constants of methane. In addition we were able to increase the accuracy of the existing centrifugal distortion constants.

\section{Introduction}

Methane belongs to the most interesting molecules in microwave spectroscopy. This is partly due to its high symmetry and its extremely small centrifugal induced dipole moment. It is also of considerable astrophysical interest. This induced us to look for transitions in microwave regions which are not covered by previous measurements [1]. We were especially interested to resolve the hyperfine structure of the F-type species caused by spin-rotation (s.-r.) and spin-spin (s.-s.) interaction which is in the order of $100 \mathrm{kHz}$ or below. This provides a method for the determination of s.-r. and s.-s. interaction constants independent of avoided-crossing molecular beam measurements [2]. The analysis of the hyperfine structure is also necessary to determine the exact center frequencies which should be used for an accurate centrifugal distortion analysis.

\section{Experimental}

All experiments were carried out on an MWFTspectrometer in the $J$-band region $(5.4$ to $8 \mathrm{GHz}$ ) at room temperature and at a pressure in the range of 0.4 to $0.5 \mathrm{~Pa}$ ( 3 to $4 \mathrm{mTorr}$ ). The polarizing pulses had a length of $750 \mathrm{~ns}$ and a pulse power of up to $20 \mathrm{~W}$ according to the maximum output power of our microwave amplifier (TWTA). For each measurement up to $3.3 \cdot 10^{7}$ single experiments with sample intervals of $10 \mathrm{~ns}$ and up to 4096 data points were averaged. The

Reprint requests to Dr. W. Stahl, Abteilung Chemische Physik im Institut für Physikalische Chemie der Universität Kiel, Olshausenstr. 40-60, D-2300 Kiel. methane sample was commercially available from Messer-Griesheim with a stated purity of $99.995 \%$. It was used without further purification.

\section{Results and Discussion \\ 1. Determination of Transition Frequencies}

In order to decrease statistical deviations all measurements were done at least three times at different polarizing frequencies. The Fourier transform algorithm was used only to get a first survey of the spectrum. Two examples are given in Figs. 1 and 2. More accurate frequencies were obtained by least squares fits of the time domain signals. This method significantly increases the accuracy especially in the case of narrow splittings [3, 4]. This also holds for splittings between a molecular signal and a perturbation frequency of non-molecular origin.

\section{Analysis of the Hyperfine Structure of F-Type Transitions}

The tensorial s.-r. and s.-s. interaction constants $c_{\mathrm{d}}$ and $d$ were obtained from a fit of all completely resolved F-type transitions. The underlying theoretical treatment of the s.-r. and s.-s. interaction of methane is given in [5]. It is based on the Hamiltonian

$$
H=H_{\mathrm{dist}}^{\mathrm{t}}+H_{\mathrm{sr}}^{\mathrm{s}}+H_{\mathrm{sr}}^{\mathrm{t}}+H_{\mathrm{ss}}
$$

with the scalar and tensorial s.-r. interaction terms $H_{\mathrm{sr}}^{\mathrm{s}}$ and $H_{\mathrm{sr}}^{\mathrm{t}}$, the s.-s. interaction term $H_{\mathrm{ss}}$, and the tensorial centrifugal distortion term

$$
H_{\mathrm{dist}}^{\mathrm{t}}=D_{\mathrm{t}} \Omega_{4} \text {. }
$$

0932-0784/89/0700-0651 \$ 01.30/0. - Please order a reprint rather than making your own copy.

Dieses Werk wurde im Jahr 2013 vom Verlag Zeitschrift für Naturforschung in Zusammenarbeit mit der Max-Planck-Gesellschaft zur Förderung der Wissenschaften e.V. digitalisiert und unter folgender Lizenz veröffentlicht: Creative Commons Namensnennung-Keine Bearbeitung 3.0 Deutschland Lizenz.
This work has been digitalized and published in 2013 by Verlag Zeitschrif für Naturforschung in cooperation with the Max Planck Society for the Advancement of Science under a Creative Commons Attribution-NoDerivs 3.0 Germany License. 


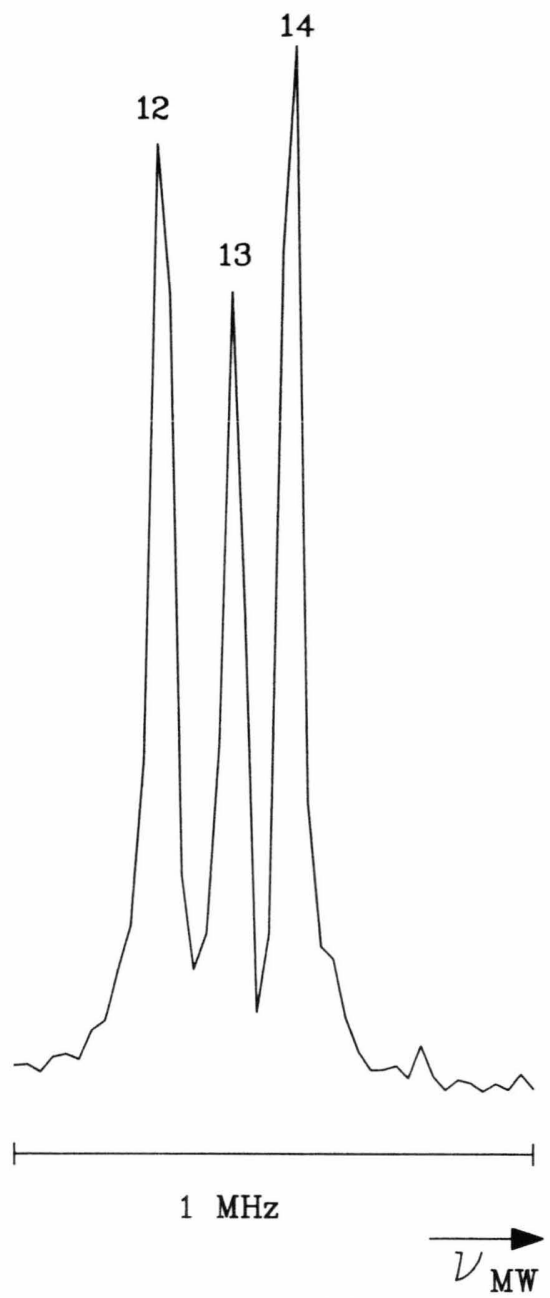

Fig. 1. $1 \mathrm{MHz}$ section of the power spectrum of methane, $J, \tau: 10, \mathrm{~A}_{1}^{(1)}-10, \mathrm{~A}_{2}^{(1)}$. Peak frequency $5014.097 \mathrm{MHz}$ (time domain signal fitted by least squares method), polarizing frequency $5013.496 \mathrm{MHz}$, pulse power ca. $20 \mathrm{~W}$, pulse length $750 \mathrm{~ns}$, sample interval $10 \mathrm{~ns}, 4096$ data points, $6.6 \cdot 10^{6}$ averaging cycles. Pressure $0.37 \mathrm{~Pa}$ (2.80 mTorr), temperature $296 \mathrm{~K}$.

Fig. 2. $1 \mathrm{MHz}$ section of the power spectrum of methane, $J, \tau: 13, \mathrm{~F}_{1}^{(4)}-13, \mathrm{~F}_{2}^{(2)}$. The components of the hyperfine structure are labelled by the quantum number $F$ of the total angular momentum. Peak frequencies $\mathrm{F}$ : 12-12: $5558.2522 \mathrm{MHz}, \mathrm{F}: 13-13: 5558.4059 \mathrm{MHz}, \mathrm{F}: 14-14$ : $5558.4993 \mathrm{MHz}$ (time domain signals fitted by least squares method), polarizing frequency $5557.996 \mathrm{MHz}$, pulse power ca. $20 \mathrm{~W}$, pulse length $750 \mathrm{~ns}$, sample interval $10 \mathrm{~ns}$, 2048 data points, filled with 2048 zeros prior to Fourier transformation, $3.3 \cdot 10^{7}$ averaging cylces. Pressure $0.48 \mathrm{~Pa}$ (3.60 mTorr), temperature $296 \mathrm{~K}$.

\begin{tabular}{|c|c|c|c|c|c|c|c|}
\hline$J$ & $\tau$ & $J^{\prime}$ & $\tau^{\prime}$ & $F^{\prime}$ & $v_{\mathrm{obs}}$ & $v_{\text {calc }}$ & $v_{\text {obs }}-v_{\text {calc }}$ \\
\hline 11 & $\mathrm{~F}_{1}^{(3)}$ & $10-11$ & $\mathrm{~F}_{2}^{(2)}$ & 10 & 5170.8096 & 5170.8116 & -2.0 \\
\hline 11 & $F_{1}^{(3)}$ & $11-11$ & $\mathrm{~F}_{2}^{(2)}$ & 11 & 5170.8762 & 5170.8752 & 1.0 \\
\hline 11 & $F_{1}^{(3)}$ & $12-11$ & $\mathrm{~F}_{2}^{(2)}$ & 12 & 5170.9378 & 5170.9368 & 1.0 \\
\hline 13 & $\mathrm{~F}_{1}^{(4)}$ & $12-13$ & $\mathrm{~F}_{2}^{(2)}$ & 12 & 5558.2522 & 5558.2520 & 0.2 \\
\hline 13 & $\mathrm{~F}_{1}^{(4)}$ & $13-13$ & $\mathrm{~F}_{2}^{(2)}$ & 13 & 5558.4059 & 5558.4061 & -0.2 \\
\hline 13 & $\mathrm{~F}_{1}^{(4)}$ & $14-13$ & $\mathrm{~F}_{2}^{(2)}$ & 14 & 5558.4993 & 5558.4993 & 0.0 \\
\hline 13 & $\mathrm{~F}_{2}^{(2)}$ & $12-13$ & $\mathrm{~F}_{1}^{(2)}$ & 12 & 6009.8425 & 6009.8434 & -0.9 \\
\hline 13 & $\mathrm{~F}_{2}^{(2)}$ & $13-13$ & $\mathrm{~F}_{1}^{(2)}$ & 13 & 6009.7155 & 6009.7157 & -0.2 \\
\hline 13 & $\mathrm{~F}_{2}^{(2)}$ & $14-13$ & $\mathrm{~F}_{1}^{(2)}$ & 14 & 6009.6777 & 6009.6766 & 1.1 \\
\hline
\end{tabular}

Table 1. Data set used for the fit of $c_{\mathrm{d}}$ and $d$. $J, J^{\prime}$ : rotational quantum number,

$\tau, \tau^{\prime}$ : symmetry species,

$F, F^{\prime}$ : quantum number of total angular momentum,

$v_{\mathrm{obs}}$ : observed frequency [MīHz],

$v_{\text {calc }}:$ calculated frequency $[\mathrm{MHz}]$ with $c_{\mathrm{d}}=18.516 \mathrm{kHz}$ and $d=21.66 \mathrm{kHz}$, $v_{\text {obs }}-v_{\text {calc }}[\mathrm{kHz}]$. 
Table 2. Fitted s.-r., s.-s. interaction constants $c_{\mathrm{d}}$ and $d$ and center frequencies. $c_{\mathrm{d}}[\mathrm{kHz}], d[\mathrm{kHz}]$, center frequencies $[\mathrm{MHz}]$. Standard deviations in units of the last significant figure in brackets.

Column 1: result with data from Table 1.

Column 2: result from avoided-crossing measurements [2]. Column 3: theoretical value [7].

\begin{tabular}{|c|c|c|c|}
\hline & 1 & 2 & 3 \\
\hline $\begin{array}{l}c_{\mathrm{d}} \\
d \\
v\left(11 \mathrm{~F}_{1}^{(3)}-\mathrm{F}_{2}^{(2)}\right) \\
v\left(13 \mathrm{~F}_{1}^{(4)}-\mathrm{F}_{2}^{(2)}\right) \\
v\left(13 \mathrm{~F}_{2}^{(2)}-\mathrm{F}_{1}^{(2)}\right)\end{array}$ & $\begin{array}{l}18.516 \quad(116) \\
21.66 \quad(64) \\
5170.8782(8) \\
5558.3919(8) \\
6009.7411(8)\end{array}$ & $\begin{array}{l}18.370(23) \\
21.17 \quad(32)\end{array}$ & 21.24 \\
\hline
\end{tabular}

Table 3. Correlation matrix of the fit of $c_{\mathrm{d}}, d$, and center frequencies.

\begin{tabular}{lrrrrr}
\hline$c_{\mathrm{d}}$ & 1.000 & & & & \\
$d$ & -0.038 & 1.000 & & & \\
$v\left(11 \mathrm{~F}_{1}^{(3)}-\mathrm{F}_{2}^{(2)}\right)$ & -0.031 & -0.002 & 1.000 & & \\
$v\left(13 \mathrm{~F}_{1}^{(4)}-\mathrm{F}_{2}^{(2)}\right)$ & 0.046 & 0.001 & -0.001 & 1.000 & \\
$v\left(13 \mathrm{~F}_{2}^{(2)}-\mathrm{F}_{1}^{(2)}\right)$ & -0.028 & -0.001 & -0.001 & 0.001 & 1.000 \\
\hline
\end{tabular}

Table 4. Complete list of measured $J$ band $Q$-branch transitions. $J$ : rotational quantum number of upper and lower level; $\tau, \tau^{\prime}$ : symmetry species of upper resp. lower level; $v_{\mathrm{obs}}$ : observed frequency $[\mathrm{MHz}] ; v_{\text {calc }}$ : calculated frequencies $[\mathrm{MHz}]$ with centrifugal distortion constants from Table 5, column $2 ; v_{\text {obs }}-v_{\text {calc }}[\mathrm{kHz}]$.

\begin{tabular}{|c|c|c|c|c|}
\hline$J$ & $\tau \quad \tau^{\prime}$ & $v_{\text {obs }}$ & $v_{\text {calc }}$ & $v_{\mathrm{obs}}-v_{\mathrm{calc}}$ \\
\hline 10 & $A_{1}^{(1)}-A_{2}^{(1)}$ & 5014.0970 & 5014.0976 & -0.6 \\
\hline 10 & $E^{(2)}-E^{(1)}$ & 6614.9000 & 6614.8997 & 0.3 \\
\hline 10 & $\mathrm{~F}_{1}^{(2)}-\mathrm{F}_{2}^{(1)}$ & 6209.5690 & 6209.5682 & 0.8 \\
\hline 11 & $E^{(2)}-E^{(1)}$ & 4600.3490 & 4600.3478 & 1.2 \\
\hline 11 & $\mathrm{~F}_{1}^{(3)}-\mathrm{F}_{2}^{(2)}$ & 5170.8780 & 5170.8807 & -2.7 \\
\hline 13 & $\mathrm{~A}_{2}^{(1)}-\mathrm{A}_{1}^{(1)}$ & 6935.0950 & 6935.0947 & 0.3 \\
\hline 13 & $\mathrm{~F}_{2}^{(2)}-\mathrm{F}_{1}^{(2)}$ & 6009.7410 & 6009.7413 & -0.3 \\
\hline 13 & $\mathrm{~F}_{1}^{(4)}-\mathrm{F}_{2}^{(2)}$ & 5558.3920 & 5558.3908 & 1.2 \\
\hline 14 & $E^{(3)}-E^{(2)}$ & 7861.6550 & 7861.6554 & -0.4 \\
\hline
\end{tabular}

$\Omega_{4}$ is the quartic centrifugal distortion operator defined in [6], $D_{\mathrm{t}}$ the corresponding quartic tensorial centrifugal distortion constant.

The high accuracy of our hyperfine data made it necessary to consider also the sextic centrifugal distortion operator $\Omega_{6}[6]$ and to replace $H_{\mathrm{dist}}^{\mathrm{t}}$ by

$$
H_{\mathrm{dist}}^{\mathrm{t} \prime}=D_{\mathrm{t}} \Omega_{4}+H_{4 \mathrm{t}} J^{2} \Omega_{4}+H_{6 \mathrm{t}} \Omega_{6},
$$

with the sextic tensorial centrifugal distortion constants $H_{4 \mathrm{t}}$ and $H_{6 \mathrm{t}}$. For the fit of the s.-r. and s.-s. interaction constants we fixed the centrifugal distortion data at $D_{\mathrm{t}}=132.943 \mathrm{kHz}, \quad H_{4 \mathrm{t}}=-16.983 \mathrm{~Hz}$, $H_{6 \mathrm{t}}=11.026 \mathrm{~Hz}$.

The frequencies of the triplet components as well as the (hypothetical) center frequencies obtained from the fit are given in Table 1. All lines were equally weighted. $c_{\mathrm{d}}, d$ and the center frequencies were treated as independent fit parameters. The results and the corresponding correlation matrix are given in Tables 2 and 3. For comparison we also included the values of $c_{\mathrm{d}}$ and $d$ measured by Itano and Ozier [2] with avoided-crossing molecular beam spectroscopy. Obviously both methods yield the same s.-r. and s.-s. interaction constants within standard errors. The s.-s. interaction constant $d$ may also be compared with the theoretical value $d=21.24 \mathrm{kHz}$ given in [7].

The scalar s.-r. constant $c_{\mathrm{a}}$ is not available from $Q$-branch transitions.

\section{Centrifugal Distortion Analysis}

All A- and E-type transition frequencies as well as the center frequencies of the F-type triplets are compiled in Table 4. In the case of not completely resolved F-type transitions we estimated the center frequency. The uncertainties were assumed to be $3 \mathrm{kHz}$ for all frequencies.

Table 5. Tensorial centrifugal distortion constants [MHz]. Standard deviations in units of the last significant figure in brackets.

Column 1: fit of transitions from Table 4.

Column 2: fit of transitions from Table 4 and Table I of [1].

Column 3: fit of transitions from Table I of [1].

All lines were weighted according to the inverse of their squared uncertainties.

\begin{tabular}{|c|c|c|c|}
\hline & 1 & 2 & 3 \\
\hline$D_{\mathrm{t}}$ & $132.94486(74) \cdot 10^{-3}$ & $132.94293(22) \cdot 10^{-3}$ & $132.94357(97) \cdot 10^{-3}$ \\
\hline $\mathrm{H}_{4 \mathrm{t}}$ & $-17.0097(103) \cdot 10^{-6}$ & $-16.9827(28) \cdot 10^{-6}$ & $-16.9790(90) \cdot 10^{-6}$ \\
\hline$H_{6 t}$ & $11.0325(28) \cdot 10^{-6}$ & $11.0261(14) \cdot 10^{-6}$ & $10.9956(38) \cdot 10^{-6}$ \\
\hline$L_{4 \mathrm{t}}$ & $2.127 \quad(34) \cdot 10^{-9}$ & $2.039 \quad(9) \cdot 10^{-9}$ & $2.000 \quad(23) \cdot 10^{-9}$ \\
\hline$L_{6 \mathrm{t}}$ & $-2.691 \quad(15) \cdot 10^{-9}$ & $-2.654 \quad(8) \cdot 10^{-9}$ & $-2.519 \quad(15) \cdot 10^{-9}$ \\
\hline$L_{8 \mathrm{t}}$ & $-2.979 \quad(57) \cdot 10^{-9}$ & $-3.009 \quad(44) \cdot 10^{-9}$ & $-2.617 \quad(92) \cdot 10^{-9}$ \\
\hline
\end{tabular}


Table 6. Correlation matrices for fits of centrifugal distortion parameters.

a) fit of transitions from Table 4

$\begin{array}{ll}D_{\mathrm{t}} & 1.000\end{array}$

$\begin{array}{lll}H_{4} & -0.997 & 1.000\end{array}$

$\begin{array}{llll}H_{6 \mathrm{t}} & 0.790 & -0.813 & 1.000\end{array}$

$\begin{array}{llll}L_{4 \mathrm{t}} & 0.982 & -0.993 & 0.841\end{array}$

$\begin{array}{llllll}L_{6 t} & -0.807 & 0.831 & -0.997 & -0.859\end{array}$

b) fit of transitions from Table 4 and Table I of [1]

D $\quad 1.000$

$H_{4 \mathrm{t}} \quad-0.980 \quad 1.000$

$H_{61}^{41} \quad 0.044-0.115$

$\begin{array}{llll}L_{4 \mathrm{l}} & 0.864 & -0.941 & 0.244\end{array}$

$\begin{array}{lllll}L_{6+} & -0.041 & 0.118 & -0.991 & -0.258\end{array}$

$\begin{array}{llllll}0.318 & -0.194 & -0.301 & -0.118 & 0.317 & 1.000\end{array}$

c) fit of transitions from Table I of [1]

$D_{1} \quad 1.000$

$\begin{array}{lrr}H_{4} & -0.969 & 1.000\end{array}$

$\begin{array}{llll}H_{6 \mathrm{t}} & -0.024 & -0.123 & 1.000\end{array}$

$\begin{array}{llll}L_{4 \mathrm{l}} & 0.845 & -0.948 & 0.327\end{array}$

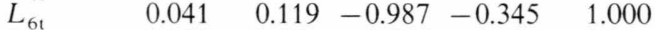

$\begin{array}{llllllll}L_{8 \mathrm{t}} & & -0.168 & 0.371 & -0.757 & -0.631 & -0.817 & 1.000\end{array}$

It is interesting to investigate whether this small amount of high precision data is sufficient to determine a complete set of quartic, sextic and octic tensorial centrifugal distortion constants. We made a fit with the programs XY4TOP and XY4FIT [8]. These programs have also been used in [1]. In this case frequencies were equally weighted. The result is presented in Table 5, column 1. For comparison we give

[1] M. Oldani, M. Andrist, A. Bauder, and A. G. Robiette, J. Mol. Spectrosc. 110, 93 (1985).

[2] W. Itano and I. Ozier, J. Chem. Phys. 72, 3700 (1980).

[3] I. Merke and H. Dreizler, Z. Naturforsch. 43a, 196 (1988).

[4] J. Haekel and H. Mäder, Z. Naturforsch. 43a, 203 (1988).

[5] J. T. Hougen, J. Mol. Spectrosc. 46, 490 (1973). the constants obtained by Oldani et al. [1] in column 3. The correlation matrices for both fits are shown in Tables $6 \mathrm{a}$ and $\mathrm{c}$. Obviously some correlation constants for our data are rather high. Therefore we decided to combine both data sets in a second fit. The results are also shown in Table 5, column 2, resp. Table $6 \mathrm{~b}$. In this fit all lines were weighted according to the inverse of their squared uncertainties. Whenever the correlation matrix is different from that of Oldani's fit, it has not become significantly worse. The error limits of the centrifugal distortion constants are only half as large now. It is remarkable that not all centrifugal distortion constants of the different fits agree within the standard error limits. We assume that this effect could arise from an influence of some 10th order constants.

\section{Acknowledgements}

The authors wish to thank the members of the Kiel microwave group for help and discussions. We also thank the Deutsche Forschungsgemeinschaft, the Fonds der Chemischen Industrie and the Land Schleswig-Holstein for funds. We are especially indebted to Dr. Jon T. Hougen for providing us with his program to calculate the s.-r. and s.-s. interaction and to Dr. I. Ozier for a discussion concerning the inclusion of the sextic centrifugal distortion terms in the hyperfine calculation.

[6] I. Ozier, J. Mol. Spectrosc. 53, 336 (1974).

[7] I. Ozier, L. M. Crapo, and S. S. Lee, Phys. Rev. 172, 63 (1968).

[8] The programs XY4TOP and XY4FIT were originally written by A. G. Robiette. 